\title{
A Study on Heterosis in Tomato (Solanum lycopersicum L.) for Yield and its Component Traits
}

\author{
Parvendra Kumar ${ }^{1}$, Neeraj Singh ${ }^{2} *$ and Pradip Kumar Singh ${ }^{1}$ \\ ${ }^{1}$ Department of Vegetable Science, N.D.U.A. \& T. Kumarganj (Faizabad), \\ Uttar Pradesh, India, (224229) \\ ${ }^{2}$ Department of Vegetable Science, G.B.P.U.A. \& T. Pantnagar (U.S. Nagar), \\ Uttarakhand-263145, India \\ *Corresponding author
}

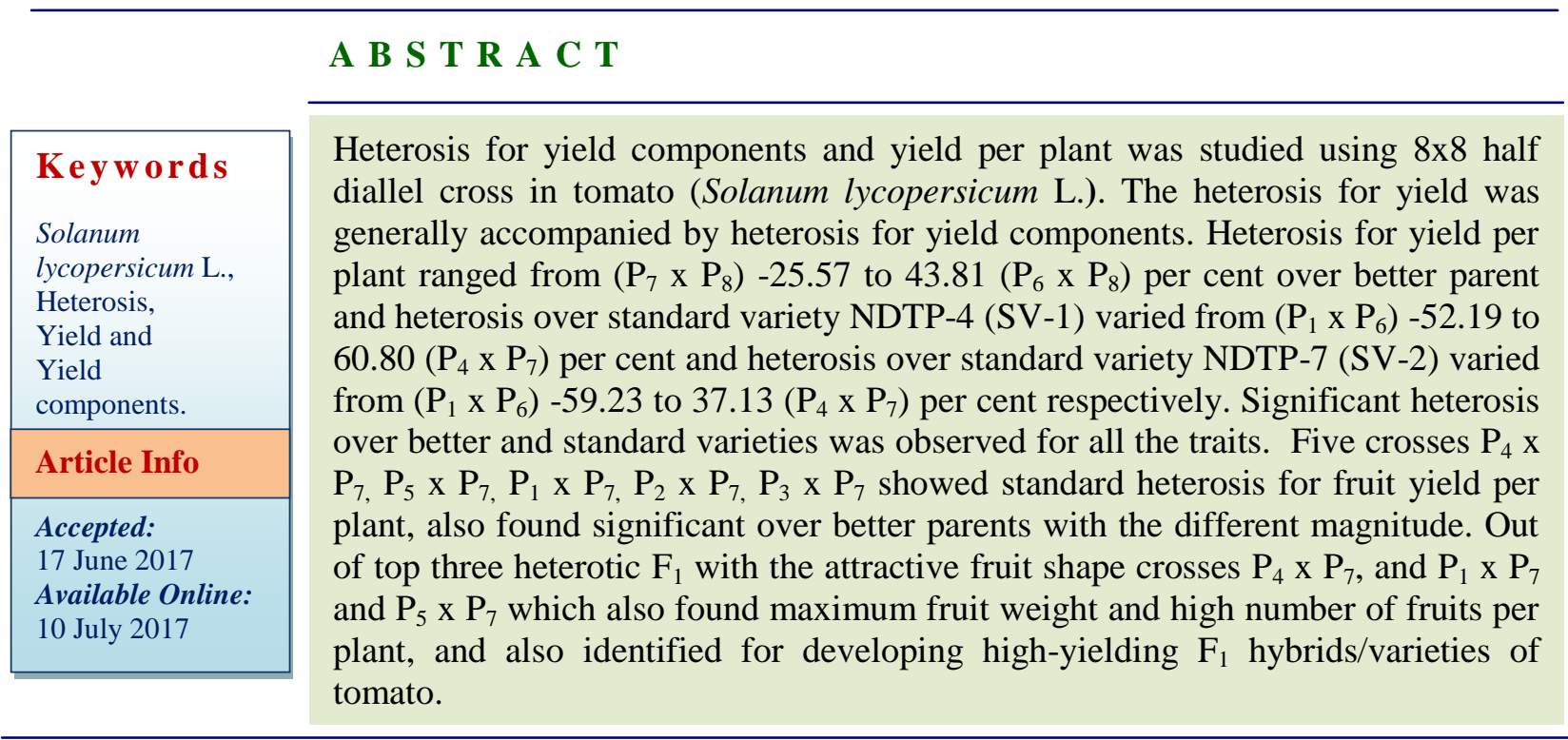

\section{Introduction}

Tomato is a popular vegetable crop in among the vegetables. It is commercial significance increased owing to the awareness about its nutritional and medicinal value, and has a consequence demand round the year among the consumers. The major objective of tomato breeding is to be developed high yielding varieties with earliness, desirable/attractive fruit shape, size, colour and free from various diseases. Heterosis breeding offers the most efficient tool to achieve this objective. Tomato being predominantly bisexual self- pollinated crop, does not suffer from inbreeding depression (Allard, 1960) and has the advantage of producing a large number of seeds per fruit, facilitating heterosis breeding through reasonably low cost of hybrid seed production. With the use of pure line in selfpollinated vegetable crop like tomato, hybrids with uniform fruits and high yielding potential can be developed to enhance productivity and production. Various breeding techniques have been advocated considering the breeding behaviour of crop species. Out of 
these hybrids breeding is prominent and used in the improvement of vegetable crops. Heterosis in tomato was first observed by Hedrick and Booth (1968) for higher yield and more number of fruits per plant. Choudhary et al., (1965) emphasized the extensive utilization of heterosis to step up tomato production. Heterosis manifestation in tomato is in the form of the greater vigour, faster growth and development, earliness in maturity, increased productivity (Yordanov, 1983). So a speedy improvement can be brought about by exploiting heterosis for various yield contributing traits as well as earliness.

\section{Materials and Methods}

The experimental material consisted of eight parental lines of tomato viz., NDTP-1 $\left(\mathrm{P}_{1}\right)$, NDTP-2 $\left(\mathrm{P}_{2}\right)$, NDTP-3 ( $\left.\mathrm{P}_{3}\right)$, NDTP-4 $\left(\mathrm{P}_{4}\right)$, NDTP-5 $\left(\mathrm{P}_{5}\right)$, NDTP-6 $\left(\mathrm{P}_{6}\right)$, NDTP-7 $\left(\mathrm{P}_{7}\right)$ and NDTP-8 $\left(\mathrm{P}_{8}\right)$. These eight parental lines were crossed in all possible combinations, excluding reciprocals to get $28 \mathrm{~F}_{1}$ 's hybrids. All the 36 genotypes (eight parental lines and 28 F's hybrids) were evaluated in Randomized Block Design (RBD) with three replications. All the agronomic practices were adopted to raise a good crop. The data were recorded on eleven quantitative characters viz., days to 50 per cent flowering, plant height $(\mathrm{cm})$, number of primary branches per plant, number of fruits per plant, average fruit weight $(\mathrm{g})$, fruit circumference $(\mathrm{cm})$, pericarp thickness $(\mathrm{mm})$, number of locules per plant, total soluble solid (TSS), fruit length $(\mathrm{cm})$ and fruit yield per plant $(\mathrm{kg})$. The mean data obtained for quantitative traits were analyzed statistically for heterosis as suggested by Fonseca and Patterson.

\section{Results and Discussion}

There were significant differences among the parental lines with respect to different characters studied including yield per plant. The mean performance of eight parental lines along with $28 \mathrm{~F} 1$ hybrids is given in table 1 . With respect to days to 50 per cent flowering range of heterosis the extent of heterosis ranged from $-10.49\left(\mathrm{P}_{4} \times \mathrm{P}_{6}\right)$ to 6.79 per cent $\left(\mathrm{P}_{1} \times \mathrm{P}_{6}\right)$. The heterosis over standard variety (SV-1) varied from $-41.43\left(\mathrm{P}_{2} \times \mathrm{P}_{8}\right)$ to 35.58 per cent $\left(\mathrm{P}_{4} \times \mathrm{P}_{7}\right)$ and heterosis over standard variety $(\mathrm{SV}-2)$ varied from $-7.66\left(\mathrm{P}_{4} \times \mathrm{P}_{8}\right)$ to 12.47 per cent $\left(\mathrm{P}_{1} \times \mathrm{P}_{6}\right)$. Out of $28 \mathrm{~F}_{1}$ hybrids, significant and desirable heterosis was observed in ten hybrids over the better parent. In accordance with the present finding, Singh and Singh (1993), Baishya et al., (2001) and Joshi and Thakur (2003) also observed earliness in heterotic combinations of tomato. The heterosis for plant height ranged from $23.47\left(\mathrm{P}_{4} \times \mathrm{P}_{6}\right)$ to 39.76 per cent $\left(\mathrm{P}_{7} \times \mathrm{P}_{8}\right)$ over better parent. Regarding standard heterosis, it ranged from $-46.68\left(\mathrm{P}_{2} \times \mathrm{P}_{3}\right)$ to 17.77 per cent $\left(\mathrm{P}_{5} \times \mathrm{P}_{7}\right)$ over standard variety $(\mathrm{SV}-1)$ and from $125.99\left(\mathrm{P}_{5} \times \mathrm{P}_{7}\right)$ to 112.34 per cent $\left(\mathrm{P}_{4} \times \mathrm{P}_{7}\right)$ over standard variety $(\mathrm{SV}$ 2 ). Out of $28 \mathrm{~F}_{1}$ 's fourteen hybrids showed heterosis in desirable direction over better parent. Similar observations were also made by Joshi and Thakur (2003), Baishya et al., (2001), Kumar et al., (2012) and Dubey et al., (2014) with a different set of material in tomato. The extent of heterosis for number of primary branches per plant varied from -20.26 $\left(\mathrm{P}_{3} \times \mathrm{P}_{5}\right)$ to 61.47 per cent $\left(\mathrm{P}_{1} \times \mathrm{P}_{2}\right)$. Heterosis over the standard variety $(\mathrm{SV}-1)$ were from $28.19\left(\mathrm{P}_{1} \times \mathrm{P}_{3}\right)$ to 65.01 per cent $\left(\mathrm{P}_{1} \times \mathrm{P}_{2}\right)$ and heterosis over the standard variety (SV-2) ranged from $-22.00\left(\mathrm{P}_{1} \times \mathrm{P}_{3}\right)$ to 79.23 per cent $\left(\begin{array}{lll}\mathrm{P}_{1} & \mathrm{x} & \mathrm{P}_{2}\end{array}\right)$. The desirable and significant heterosis was observed by six crosses over better parent while, six crosses showed positive significant and desirable heterosis over both the standard varieties. These results are in consonance with Sundaram et al., (1994), Baishya et al., (2001) and Garg and Cheema (2010) in tomato. 
Table.1 Mean value for yield and yield attributing traits of parents and the $F_{1}$ hybrids

\begin{tabular}{|c|c|c|c|c|c|c|c|c|c|c|c|}
\hline Genotype & $\begin{array}{c}\text { Days to 50\% } \\
\text { flowering }\end{array}$ & $\begin{array}{c}\text { Plant } \\
\text { height } \\
(\mathbf{c m})\end{array}$ & $\begin{array}{l}\text { No. of primary } \\
\text { branches/ plant }\end{array}$ & $\begin{array}{l}\text { Number of } \\
\text { fruits/plant }\end{array}$ & $\begin{array}{l}\text { Average fruit } \\
\text { Weight(g) }\end{array}$ & $\begin{array}{c}\text { Fruitcircumf } \\
\text { erence }(\mathrm{cm})\end{array}$ & $\begin{array}{c}\text { Pericarp } \\
\text { thickness } \\
(\mathbf{m m})\end{array}$ & $\begin{array}{c}\text { Number of } \\
\text { locules/Fruit }\end{array}$ & $\begin{array}{c}\text { Total } \\
\text { soluble } \\
\text { Solid (\%) }\end{array}$ & $\begin{array}{c}\text { Fruit } \\
\text { length }(\mathrm{cm})\end{array}$ & $\begin{array}{c}\text { Fruit } \\
\text { yield/plant }\end{array}$ \\
\hline NDTP-1 $\left(\mathrm{P}_{1}\right)$ & 58.00 & 86.52 & 3.82 & 19.07 & 30.35 & 15.16 & 4.84 & 4.82 & 5.29 & 7.90 & 0.53 \\
\hline NDTP-2 $\left(\mathrm{P}_{2}\right)$ & 57.67 & 66.45 & 4.81 & 16.38 & 46.63 & 17.86 & 4.24 & 5.15 & 4.45 & 7.49 & 0.69 \\
\hline NDTP-3 $\left(\mathrm{P}_{3}\right)$ & 56.00 & 57.44 & 3.29 & 19.87 & 33.66 & 15.54 & 3.63 & 3.37 & 4.20 & 7.49 & 0.61 \\
\hline NDTP-4 $\left(\mathrm{P}_{4}\right)$ & 53.67 & 113.86 & 4.71 & 23.60 & 57.21 & 17.89 & 3.97 & 6.46 & 6.35 & 7.94 & 1.23 \\
\hline NDTP-5 $\left(\mathrm{P}_{5}\right)$ & 58.33 & 96.43 & 5.12 & 18.18 & 68.65 & 15.28 & 4.61 & 4.75 & 4.53 & 6.79 & 1.12 \\
\hline NDTP-6 $\left(\mathrm{P}_{6}\right)$ & 59.33 & 67.33 & 4.43 & 16.06 & 36.30 & 14.30 & 3.63 & 4.16 & 4.32 & 6.67 & 0.53 \\
\hline NDTP-7 $\left(\mathrm{P}_{7}\right)$ & 56.33 & 59.33 & 4.33 & 25.22 & 62.74 & 15.68 & 3.85 & 4.61 & 4.61 & 7.26 & 1.44 \\
\hline $\mathrm{P}_{1} \times \mathrm{P}_{2}$ & 60.73 & 84.13 & 7.77 & 21.27 & 36.29 & 17.33 & 4.67 & 5.23 & 5.11 & 8.08 & 0.70 \\
\hline $\mathrm{P}_{1} \times \mathrm{P}_{3}$ & 54.72 & 67.66 & 3.38 & 19.08 & 39.22 & 14.58 & 4.15 & 4.39 & 4.60 & 7.38 & 0.68 \\
\hline $\mathrm{P}_{1} \times \mathrm{P}_{4}$ & 56.95 & 115.22 & 4.69 & 27.74 & 51.61 & 17.18 & 4.67 & 5.69 & 6.17 & 8.08 & 1.33 \\
\hline $\mathrm{P}_{1} \times \mathrm{P}_{5}$ & 55.84 & 87.82 & 4.25 & 18.25 & 60.03 & 14.91 & 4.68 & 5.25 & 4.72 & 7.05 & 1.00 \\
\hline $\mathrm{P}_{1} \times \mathrm{P}_{6}$ & 63.36 & 92.31 & 4.95 & 18.80 & 34.30 & 16.20 & 4.66 & 4.61 & 4.95 & 7.87 & 0.59 \\
\hline $\mathrm{P}_{1} \times \mathrm{P}_{7}$ & 55.45 & 67.09 & 4.28 & 29.24 & 56.11 & 16.03 & 4.52 & 5.37 & 4.80 & 7.35 & 1.51 \\
\hline $\mathrm{P}_{1} \times \mathrm{P}_{8}$ & 61.17 & 96.43 & 4.29 & 23.34 & 33.82 & 16.16 & 4.36 & 4.79 & 5.49 & 7.66 & 0.72 \\
\hline $\mathrm{P}_{2} \times \mathrm{P}_{3}$ & 53.42 & 60.71 & 4.01 & 17.40 & 45.26 & 15.03 & 3.82 & 5.30 & 4.24 & 7.04 & 0.72 \\
\hline $\mathrm{P}_{2} \times \mathrm{P}_{4}$ & 57.34 & 110.89 & 5.24 & 24.19 & 60.73 & 19.31 & 4.31 & 4.76 & 5.67 & 7.95 & 1.33 \\
\hline $\mathrm{P}_{2} \times \mathrm{P}_{7}$ & 59.85 & 72.96 & 5.03 & 24.34 & 67.48 & 18.11 & 4.17 & 3.91 & 4.75 & 7.75 & 1.50 \\
\hline $\mathrm{P}_{2} \times \mathrm{P}_{8}$ & 58.14 & 80.39 & 4.82 & 22.49 & 33.50 & 16.98 & 4.21 & 3.82 & 5.14 & 7.09 & 0.68 \\
\hline $\mathrm{P}_{3} \times \mathrm{P}_{4}$ & 58.12 & 99.35 & 4.40 & 25.86 & 48.78 & 16.89 & 3.99 & 4.56 & 5.75 & 8.18 & 1.15 \\
\hline $\mathrm{P}_{3} \times \mathrm{P}_{5}$ & 56.60 & 83.86 & 4.08 & 21.68 & 43.28 & 14.79 & 4.37 & 5.88 & 4.19 & 7.07 & 0.85 \\
\hline $\mathrm{P}_{3} \times \mathrm{P}_{6}$ & 55.36 & 61.14 & 4.48 & 17.61 & 39.09 & 15.67 & 3.52 & 5.63 & 4.35 & 6.80 & 0.63 \\
\hline $\mathrm{P}_{3} \times \mathrm{P}_{7}$ & 52.24 & 68.90 & 3.66 & 25.93 & 62.95 & 16.39 & 3.70 & 5.37 & 4.27 & 6.86 & 1.50 \\
\hline $\mathrm{P}_{3} \times \mathrm{P}_{8}$ & 58.41 & 82.26 & 5.54 & 25.42 & 35.39 & 16.52 & 3.93 & 5.20 & 4.91 & 7.23 & 0.82 \\
\hline $\mathrm{P}_{4} \times \mathrm{P}_{5}$ & 61.20 & 115.83 & 7.48 & 26.92 & 59.33 & 18.67 & 4.71 & 6.26 & 5.96 & 8.57 & 1.45 \\
\hline $\mathrm{P}_{4} \times \mathrm{P}_{6}$ & 53.11 & 87.13 & 3.88 & 24.76 & 44.90 & 15.42 & 3.98 & 4.98 & 5.37 & 7.51 & 1.01 \\
\hline $\mathrm{P}_{4} \times \mathrm{P}_{7}$ & 56.41 & 125.99 & 5.40 & 27.97 & 77.56 & 19.65 & 4.54 & 5.11 & 6.50 & 8.17 & 1.97 \\
\hline $\mathrm{P}_{4} \times \mathrm{P}_{8}$ & 52.02 & 101.84 & 4.70 & 20.51 & 56.79 & 15.58 & 4.67 & 5.25 & 5.81 & 7.12 & 0.98 \\
\hline $\mathrm{P}_{5} \times \mathrm{P}_{6}$ & 60.24 & 99.09 & 6.04 & 21.26 & 44.33 & 17.00 & 4.59 & 5.05 & 4.60 & 7.25 & 0.86 \\
\hline $\mathrm{P}_{5} \times \mathrm{P}_{7}$ & 59.17 & 134.09 & 5.15 & 27.74 & 61.21 & 17.54 & 4.75 & 4.72 & 4.95 & 7.35 & 1.54 \\
\hline $\mathrm{P}_{5} \times \mathrm{P}_{8}$ & 57.36 & 99.32 & 4.61 & 23.87 & 43.40 & 14.93 & 4.31 & 4.66 & 4.91 & 6.93 & 0.94 \\
\hline $\mathrm{P}_{6} \times \mathrm{P}_{7}$ & 60.33 & 75.54 & 4.64 & 22.41 & 57.95 & 16.28 & 3.91 & 4.54 & 4.58 & 7.34 & 1.17 \\
\hline $\mathrm{P}_{6} \times \mathrm{P}_{8}$ & 56.00 & 88.22 & 4.59 & 19.52 & 67.05 & 15.13 & 3.77 & 4.93 & 5.14 & 7.02 & 1.19 \\
\hline $\mathrm{P}_{7} \times \mathrm{P}_{8}$ & 55.41 & 91.31 & 5.27 & 21.15 & 55.71 & 17.97 & 4.42 & 4.21 & 4.83 & 6.98 & 1.07 \\
\hline Grand Mean & 57.29 & $\begin{array}{l}86.81 \\
\end{array}$ & 4.76 & 22.15 & 49.71 & 16.39 & 4.23 & 4.86 & 4.99 & 7.39 & 1.02 \\
\hline C.V. & 1.95 & 1.52 & 6.12 & 1.99 & 2.49 & 1.72 & 6.80 & 5.23 & 5.66 & 2.76 & 2.70 \\
\hline
\end{tabular}


Table.2 Estimates of heterosis (\%) over better parent (BP) and standard varieties SV-1 indeterminate and SV-2 determinate type (NDTP-4 and NDTP-7) respectively

\begin{tabular}{|c|c|c|c|c|c|c|c|c|c|c|c|c|}
\hline \multirow[b]{2}{*}{ Crosses } & \multicolumn{3}{|c|}{ Days to $50 \%$ flowering } & \multicolumn{3}{|c|}{ Plant height $(\mathrm{cm})$} & \multicolumn{3}{|c|}{ No. of primary branches per plant } & \multicolumn{3}{|c|}{ Number of fruits per plant (g) } \\
\hline & $\mathrm{BP}$ & SV1 & SV2 & $\mathrm{BP}$ & SV1 & SV2 & $\mathrm{BP}$ & SV1 & SV2 & $\mathrm{BP}$ & SV1 & SV2 \\
\hline $\mathbf{P}_{1} \times \mathbf{P}_{2}$ & $4.70 * *$ & $13.16^{* * *}$ & $7.80 * *$ & $-2.75^{*}$ & $-26.11 * *$ & $41.80 * *$ & $61.47 * *$ & $65.01 * *$ & $79.23 * *$ & $11.52 * *$ & $-9.86 * *$ & $-15.67 * *$ \\
\hline $\mathbf{P}_{1} \times \mathbf{P}_{3}$ & $-5.66^{* *}$ & 1.96 & -2.86 & $-21.80 * *$ & $-40.57 * *$ & $14.03 * *$ & -11.52 & $-28.19 * *$ & $-22.00 * *$ & $-3.94 *$ & $-19.13 * *$ & $-24.34 * *$ \\
\hline $\mathbf{P}_{1} \times \mathbf{P}_{4}$ & -1.81 & $6.12 * *$ & 1.09 & 1.19 & 1.19 & $94.19 * *$ & -0.35 & -0.35 & 8.23 & $17.54 * *$ & $17.54 * *$ & $9.96^{* *}$ \\
\hline$P_{1} \times P_{5}$ & $-4.27 * *$ & $4.05^{*}$ & -0.88 & $-8.94 * *$ & $-22.87 * *$ & $48.01 * *$ & $-17.00 * *$ & -9.77 & -2.00 & $-4.30 *$ & $-22.64 * *$ & $-27.63^{* *}$ \\
\hline $\mathbf{P}_{1} \times \mathbf{P}_{6}$ & $6.79 * *$ & $18.06 * *$ & $12.47 * *$ & $6.70 * *$ & $-18.92 * *$ & $55.58 * *$ & $11.75^{*}$ & 5.10 & $14.15^{*}$ & -1.45 & $-20.34 * *$ & $-25.48 * *$ \\
\hline $\mathbf{P}_{1} \times \mathbf{P}_{7}$ & $-4.39 * *$ & 3.33 & -1.56 & $-22.45 * *$ & $-41.08 * *$ & $13.07 * *$ & -1.23 & -9.07 & -1.23 & $15.92 * *$ & $23.92 * *$ & $15.92 * *$ \\
\hline $\mathbf{P}_{1} \times \mathbf{P}_{8}$ & $5.47 * *$ & $13.99 * *$ & $8.59 * *$ & $11.45 * *$ & $-15.31 * *$ & $62.52 * *$ & 12.30 & -8.85 & -1.00 & $13.95 * *$ & -1.09 & $-7.47 * *$ \\
\hline $\mathbf{P}_{2} \times \mathbf{P}_{3}$ & $-7.36^{* *}$ & -0.45 & $-5.17 * *$ & $-8.64 * *$ & $-46.68 * *$ & 2.32 & $-16.63 * *$ & $-14.80 * *$ & -7.46 & $-12.43 * *$ & $-26.27 * *$ & $-31.03^{* *}$ \\
\hline$P_{2} \times P_{4}$ & -0.57 & $6.84 * *$ & 1.79 & $-2.61 * *$ & $-2.61 * *$ & $86.89 * *$ & 8.87 & $11.26^{*}$ & $20.85 * *$ & 2.50 & 2.50 & $-4.11 * *$ \\
\hline $\mathbf{P}_{2} \times \mathbf{P}_{5}$ & $3.41 *$ & $12.40 * *$ & $7.08 * *$ & $-3.72 * *$ & $-18.45 * *$ & $56.48 * *$ & $11.60 *$ & $21.32 * *$ & $31.77 * *$ & $19.75 * *$ & $-7.74 * *$ & $-13.69 * *$ \\
\hline$P_{2} \times P_{6}$ & $-3.38 *$ & $6.83^{* *}$ & 1.77 & $4.32 * *$ & $-38.31 * *$ & $18.38 * *$ & 3.74 & 6.02 & $15.15^{* *}$ & $10.91 * *$ & $-23.03^{* *}$ & $-27.99 * *$ \\
\hline $\mathbf{P}_{2} \times \mathbf{P}_{7}$ & $3.79 *$ & $11.53 * *$ & $6.25 * *$ & $9.79 * *$ & $-35.92 * *$ & $22.96 * *$ & 4.57 & 6.87 & $16.08 * *$ & $-3.52 *$ & $3.14 *$ & $-3.52 *$ \\
\hline$P_{2} \times P_{8}$ & 0.82 & $8.34 * *$ & 3.21 & $20.97 * *$ & $-29.39 * *$ & $35.49 * *$ & 0.14 & 2.34 & 11.15 & $9.78 * *$ & $-4.70 * *$ & $-10.85^{* *}$ \\
\hline $\mathbf{P}_{3} \times \mathbf{P}_{4}$ & $3.79 *$ & $8.30 * *$ & 3.18 & $-12.74 * *$ & $-12.74 * *$ & $67.45 * *$ & -6.59 & -6.59 & 1.46 & $9.61 * *$ & $9.61 * *$ & 2.54 \\
\hline $\mathbf{P}_{3} \times \mathbf{P}_{5}$ & -2.98 & $5.46^{* *}$ & 0.47 & $-13.03 * *$ & $-26.34 * *$ & $41.34 * *$ & $-20.26^{* *}$ & $-13.31 *$ & -5.85 & $9.14 * *$ & $-8.11 * *$ & $-14.03^{* *}$ \\
\hline $\mathbf{P}_{3} \times \mathbf{P}_{6}$ & $-6.70 * *$ & 3.16 & -1.73 & $-9.20 * *$ & $-46.30 * *$ & 3.04 & 1.13 & -4.89 & 3.31 & $-11.38 * *$ & $-25.38 * *$ & $-30.20 * *$ \\
\hline $\mathbf{P}_{3} \times \mathbf{P}_{7}$ & $-7.27 * *$ & -2.66 & $-7.27 * *$ & $16.12 * *$ & $-39.49 * *$ & $16.12 * *$ & $-15.62 * *$ & $-22.31 * *$ & $-15.62 * *$ & 2.79 & $9.87 * *$ & 2.79 \\
\hline $\mathbf{P}_{3} \times \mathbf{P}_{8}$ & $3.69 *$ & $8.84 * *$ & $3.69 *$ & $25.91 * *$ & $-27.75^{* *}$ & $38.64 * *$ & $52.29 * *$ & $17.78 * *$ & $27.92 * *$ & $24.08 * *$ & $7.71 * *$ & 0.77 \\
\hline $\mathbf{P}_{4} \times \mathbf{P}_{5}$ & $4.91 * *$ & $14.03 * *$ & $8.63 * *$ & 1.73 & 1.73 & $95.21 * *$ & $46.25 * *$ & $58.99 * *$ & $72.69 * *$ & $14.08 * *$ & $14.08 * *$ & $6.73 * *$ \\
\hline$P_{4} \times P_{6}$ & $-10.49 * *$ & -1.04 & $-5.73 * *$ & $-23.47 * *$ & $-23.47 * *$ & $46.85 * *$ & $-17.49 * *$ & $-17.49 * *$ & -10.38 & $4.92 * *$ & $4.92 * *$ & -1.85 \\
\hline $\mathbf{P}_{4} \times \mathbf{P}_{7}$ & 0.14 & $5.12 * *$ & 0.14 & $10.66^{* *}$ & $10.66^{* *}$ & $112.34 * *$ & $14.80 * *$ & $14.80 * *$ & $24.69 * *$ & $10.90 * *$ & $18.55^{* *}$ & $10.90 * *$ \\
\hline $\mathbf{P}_{4} \times \mathbf{P}_{8}$ & $-7.66^{* *}$ & -3.07 & $-7.66 * *$ & $-10.55^{* *}$ & $-10.55^{* *}$ & $71.65 * *$ & -0.14 & -0.14 & 8.46 & $-13.10^{* *}$ & $-13.10^{* *}$ & $-18.70^{* *}$ \\
\hline $\mathbf{P}_{5} \times \mathbf{P}_{6}$ & 1.53 & $12.25 * *$ & $6.93 * *$ & $2.75^{*}$ & $-12.97 * *$ & $67.01 * *$ & $17.98 * *$ & $28.26 * *$ & $39.31 * *$ & $16.96^{* *}$ & $-9.89 * *$ & $-15.70^{* *}$ \\
\hline $\mathbf{P}_{5} \times \mathbf{P}_{7}$ & 1.43 & $10.25 * *$ & $5.04 * *$ & $39.05 * *$ & $17.77 * *$ & $125.99 * *$ & 0.65 & 9.42 & $18.85^{* *}$ & $9.98 * *$ & $17.56^{* * *}$ & $9.98^{* *}$ \\
\hline $\mathbf{P}_{5} \times \mathbf{P}_{8}$ & -1.67 & $6.88 * *$ & 1.82 & $2.99 * *$ & $-12.77 * *$ & $67.39 * *$ & $-9.97 *$ & -2.12 & 6.31 & $16.55 * *$ & 1.17 & $-5.35 * *$ \\
\hline $\mathbf{P}_{6} \times \mathbf{P}_{7}$ & 1.67 & $12.41 * *$ & $7.09 * *$ & $12.19 * *$ & $-33.65 * *$ & $27.32 * *$ & 4.82 & -1.42 & 7.08 & $-11.15^{* *}$ & $-5.03 * *$ & $-11.15^{* *}$ \\
\hline $\mathbf{P}_{6} \times \mathbf{P}_{8}$ & $-5.62 * *$ & $4.35^{*}$ & -0.59 & $31.02 * *$ & $-22.52 * *$ & $48.69 * *$ & 3.69 & -2.48 & 5.92 & $-4.69 * *$ & $-17.26^{* *}$ & $-22.60 * *$ \\
\hline $\mathbf{P}_{7} \times \mathbf{P}_{8}$ & -1.64 & 3.25 & -1.64 & $39.76^{* *}$ & $-19.81 * *$ & $53.89 * *$ & $21.69 * *$ & $12.04 *$ & $21.69 * *$ & $-16.15 * *$ & $-10.37 * *$ & $-16.15^{* *}$ \\
\hline Mean & -1.05 & 6.92 & 1.86 & 3.45 & -21.19 & 51.24 & 5.75 & 4.19 & 13.17 & 4.96 & -3.33 & -9.57 \\
\hline Highest & 6.79 & 18.06 & 12.47 & 39.76 & 17.77 & 125.99 & 61.47 & 65.01 & 79.23 & 24.08 & 23.92 & 15.92 \\
\hline Lowest & -10.49 & -3.07 & -7.66 & -23.47 & -46.68 & 2.32 & -20.26 & -28.19 & -22.00 & -1.45 & -1.09 & -1.85 \\
\hline
\end{tabular}

* - Significant at 5 per cent probability level, ** - Significant at 1 per cent probability level 


\begin{tabular}{|c|c|c|c|c|c|c|c|c|c|c|c|c|}
\hline \multirow{2}{*}{ Crosses } & \multicolumn{3}{|c|}{ Total soluble solid (TSS) } & \multicolumn{3}{|c|}{ Fruit circumference $(\mathrm{cm})$} & \multicolumn{3}{|c|}{ Pericarp thickness (mm) } & \multicolumn{3}{|c|}{ No. of locules per fruit } \\
\hline & $\mathrm{BP}$ & SV1 & SV2 & $\mathrm{BP}$ & SV1 & SV2 & $\mathrm{BP}$ & SV1 & SV2 & $\mathrm{BP}$ & SV1 & SV2 \\
\hline $\mathrm{P}_{1} \times \mathrm{P}_{2}$ & -3.34 & $-19.43 * *$ & $11.00 *$ & $-2.95 *$ & $-3.13 *$ & $10.54 * *$ & -3.38 & $17.62 * *$ & $21.28 * *$ & 1.55 & $-18.95 * *$ & $13.44 * *$ \\
\hline $\mathrm{P}_{1} \times \mathrm{P}_{3}$ & $-12.98 * *$ & $-27.47 * *$ & -0.07 & $-6.18^{* *}$ & $-18.50 * *$ & $-6.99 * *$ & $-14.20 * *$ & 4.45 & 7.70 & $-8.92 *$ & $-32.01 * *$ & -4.84 \\
\hline $\mathrm{P}_{1} \times \mathrm{P}_{4}$ & -2.84 & -2.84 & $33.86 * *$ & $-3.97 * *$ & $-3.97 * *$ & $9.59 * *$ & -3.45 & $17.53 * *$ & $21.19 * *$ & $-11.93 * *$ & $-11.93 * *$ & $23.27 * *$ \\
\hline $\mathrm{P}_{1} \times \mathrm{P}_{5}$ & $-10.84 *$ & $-25.68 * *$ & 2.39 & -2.38 & $-16.65 * *$ & $-4.89 * *$ & -3.31 & $17.70 * *$ & $21.37 * *$ & $8.85^{*}$ & $-18.74 * *$ & $13.73 * *$ \\
\hline $\mathrm{P}_{1} \times \mathrm{P}_{6}$ & -6.43 & $-22.01 * *$ & 7.45 & $6.91 * *$ & $-9.44 * *$ & $3.34 *$ & -3.72 & $17.20^{* * *}$ & $20.85^{* *}$ & -4.36 & $-28.60 * *$ & -0.07 \\
\hline $\mathrm{P}_{1} \times \mathrm{P}_{7}$ & $-9.20 *$ & $-24.32 * *$ & 4.27 & 2.25 & $-10.39 * *$ & 2.25 & -6.55 & $13.76^{*}$ & $17.30 * *$ & $11.48 * *$ & $-16.78^{* *}$ & $16.47 * *$ \\
\hline $\mathrm{P}_{1} \times \mathrm{P}_{8}$ & 3.72 & $-13.55^{* *}$ & $19.10 * *$ & $6.60 * *$ & $-9.71 * *$ & $3.04 *$ & -9.86 & 9.73 & $13.15^{*}$ & -0.62 & $-25.81 * *$ & 3.83 \\
\hline $\mathrm{P}_{2} \times \mathrm{P}_{3}$ & -4.79 & $-33.25 * *$ & -8.03 & $-15.85^{* *}$ & $-16.00^{* *}$ & $-4.15 * *$ & -9.91 & -3.94 & -0.95 & 2.85 & $-17.91 * *$ & $14.88^{* *}$ \\
\hline $\mathrm{P}_{2} \times \mathrm{P}_{4}$ & $-10.66^{* *}$ & $-10.66^{* *}$ & $23.08 * *$ & $7.90 * *$ & $7.90 * *$ & $23.13^{* *}$ & 1.73 & 8.47 & 11.85 & $-26.23 * *$ & $-26.23^{* *}$ & 3.25 \\
\hline $\mathrm{P}_{2} \times \mathrm{P}_{5}$ & 3.01 & $-26.42 * *$ & 1.37 & 2.05 & 1.86 & $16.24 * *$ & 2.60 & $19.13 * *$ & $22.84 * *$ & $-17.34 * *$ & $-34.02 * *$ & -7.66 \\
\hline $\mathrm{P}_{2} \times \mathrm{P}_{6}$ & -5.39 & $-33.67 * *$ & -8.61 & $-13.57 * *$ & $-13.73 * *$ & -1.55 & -9.05 & -3.02 & 0.00 & $-21.86 * *$ & $-37.64 * *$ & $-12.72 * *$ \\
\hline $\mathrm{P}_{2} \times \mathrm{P}_{7}$ & 3.18 & $-25.11 * *$ & 3.18 & 1.42 & 1.23 & $15.52 * *$ & -1.65 & 4.87 & 8.13 & $-24.13 * *$ & $-39.44 * *$ & $-15.25 * *$ \\
\hline $\mathrm{P}_{2} \times \mathrm{P}_{8}$ & -0.39 & $-18.96 * *$ & $11.65^{*}$ & $-4.91 * *$ & $-5.09 * *$ & $8.31 * *$ & -0.55 & 6.04 & 9.34 & $-25.81 * *$ & $-40.78^{* *}$ & $-17.12^{* *}$ \\
\hline $\mathrm{P}_{3} \times \mathrm{P}_{4}$ & $-9.40 *$ & $-9.40 *$ & $24.82 * *$ & $-5.63 * *$ & $-5.63 * *$ & $7.70 * *$ & 0.50 & 0.50 & 3.63 & $-29.43 * *$ & $-29.43^{*} *$ & -1.23 \\
\hline $\mathrm{P}_{3} \times \mathrm{P}_{5}$ & -7.50 & $-33.93 * *$ & -8.97 & $-4.83 * *$ & $-17.32 * *$ & $-5.65 * *$ & -5.27 & 9.98 & $13.41^{*}$ & $23.95 * *$ & $-8.88 * *$ & $27.53 * *$ \\
\hline $\mathrm{P}_{3} \times \mathrm{P}_{6}$ & 0.62 & $-31.51 * *$ & -5.64 & 0.81 & $-12.43 * *$ & -0.06 & -3.03 & -11.33 & -8.56 & $35.26 * *$ & $-12.85^{* *}$ & $21.97 * *$ \\
\hline $\mathrm{P}_{3} \times \mathrm{P}_{7}$ & -7.31 & $-32.72 * *$ & -7.31 & $4.53^{* * *}$ & $-8.40 * *$ & $4.53 * *$ & -3.89 & -6.80 & -3.89 & $16.33 * *$ & $-16.88^{* *}$ & $16.33 * *$ \\
\hline $\mathrm{P}_{3} \times \mathrm{P}_{8}$ & -4.84 & $-22.58 * *$ & 6.66 & $6.26^{* * *}$ & $-7.69 * *$ & $5.34 * *$ & 5.83 & -1.09 & 1.99 & $28.27 * *$ & $-19.41 * *$ & $12.79 * *$ \\
\hline $\mathrm{P}_{4} \times \mathrm{P}_{5}$ & -6.14 & -6.14 & $29.31 * *$ & $4.36 * *$ & $4.36 * *$ & $19.09^{* *}$ & 2.17 & $18.62 * *$ & $22.32 * *$ & -3.10 & -3.10 & $35.62 * *$ \\
\hline $\mathrm{P}_{4} \times \mathrm{P}_{6}$ & $-15.44 * *$ & $-15.44 * *$ & $16.50 * *$ & $-13.80 * *$ & $-13.80 * *$ & -1.64 & 0.17 & 0.17 & 3.29 & $-22.92 * *$ & $-22.92 * *$ & 7.88 \\
\hline $\mathrm{P}_{4} \times \mathrm{P}_{7}$ & 2.47 & 2.47 & $41.17 * *$ & $9.80 * *$ & $9.80 * *$ & $25.30 * *$ & $14.18^{*}$ & $14.18 *$ & $17.73 * *$ & $-20.81 * *$ & $-20.81 * *$ & $10.84^{*}$ \\
\hline $\mathrm{P}_{4} \times \mathrm{P}_{8}$ & $-8.51 *$ & $-8.51 *$ & 26.05 & $-12.91 * *$ & $-12.91 * *$ & -0.62 & $17.53 * *$ & $17.53 * *$ & $21.19 * *$ & $-18.74 * *$ & $-18.74 * *$ & $13.73 * *$ \\
\hline $\mathrm{P}_{5} \times \mathrm{P}_{6}$ & 1.47 & $-27.52 * *$ & -0.14 & $11.26^{* *}$ & $-5.01 * *$ & $8.40 * *$ & -0.58 & $15.44 *$ & $19.03 * *$ & 6.46 & $-21.73 * *$ & $9.54 *$ \\
\hline $\mathrm{P}_{5} \times \mathrm{P}_{7}$ & 7.45 & $-22.01 * *$ & 7.45 & $11.84 * *$ & -1.99 & $11.84 * *$ & 2.89 & $19.46^{* * *}$ & $23.18^{* *}$ & -0.56 & $-26.90^{* *}$ & 2.31 \\
\hline $\mathrm{P}_{5} \times \mathrm{P}_{8}$ & -4.91 & $-22.64 * *$ & 6.58 & -2.27 & $-16.56^{* *}$ & $-4.78 * *$ & -6.65 & 8.39 & 11.76 & -1.90 & $-27.88 * *$ & 0.94 \\
\hline $\mathrm{P}_{6} \times \mathrm{P}_{7}$ & -0.58 & $-27.84 * *$ & -0.58 & $3.85 *$ & $-9.00 * *$ & $3.85 *$ & 1.47 & -1.59 & 1.47 & -1.52 & $-29.63 * *$ & -1.52 \\
\hline $\mathrm{P}_{6} \times \mathrm{P}_{8}$ & -0.39 & $-18.96^{* *}$ & $11.65^{*}$ & 4.39 ** & $-15.46^{* *}$ & $-3.53^{*}$ & 1.53 & -5.12 & -2.16 & $18.43 * *$ & $-23.70 * *$ & 6.79 \\
\hline $\mathrm{P}_{7} \times \mathrm{P}_{8}$ & -6.52 & $-23.95 * *$ & 4.78 & $14.60 * *$ & 0.43 & $14.60 * *$ & $14.71 *$ & 11.24 & $14.71^{*}$ & -8.67 & $-34.74 * *$ & -8.67 \\
\hline Mean & -4.16 & -20.86 & 9.03 & 0.34 & -7.40 & 5.67 & -0.70 & 7.83 & 11.18 & -3.41 & -23.80 & 6.64 \\
\hline Highest & 7.45 & 2.47 & 41.17 & 14.60 & 9.80 & 25.30 & 17.53 & 19.46 & 23.18 & 35.26 & -3.10 & 35.62 \\
\hline Lowest & -15.44 & -33.93 & -8.97 & -15.85 & -18.50 & -6.99 & -14.20 & -11.30 & -8.50 & -29.43 & -48.78 & -17.12 \\
\hline
\end{tabular}




\begin{tabular}{|c|c|c|c|c|c|c|c|c|c|}
\hline \multirow{2}{*}{ Crosses } & \multicolumn{3}{|c|}{ Fruit length $(\mathrm{cm})$} & \multicolumn{3}{|c|}{ Fruit yield per plant (kg) } & \multicolumn{3}{|c|}{ Average fruit weight (g) } \\
\hline & $\mathrm{BP}$ & SV1 & SV2 & $\mathrm{BP}$ & SV1 & SV2 & $\mathrm{BP}$ & SV1 & SV2 \\
\hline $\mathrm{P}_{1} \times \mathrm{P}_{2}$ & 2.28 & 1.72 & $11.20 * *$ & 1.20 & $\begin{array}{c}- \\
42.76^{* *}\end{array}$ & $\begin{array}{c}- \\
51.18 * *\end{array}$ & $\begin{array}{c}- \\
22.17 * *\end{array}$ & $\begin{array}{c}- \\
36.56 * *\end{array}$ & $42.16^{* *}$ \\
\hline $\mathrm{P}_{1} \times \mathrm{P}_{3}$ & $-6.50 * *$ & $-7.01 * *$ & 1.65 & $12.09 * *$ & $\begin{array}{c}- \\
44.55^{* *} \\
\end{array}$ & $\begin{array}{c}- \\
52.71 * * \\
\end{array}$ & $16.52 * *$ & $\begin{array}{c}- \\
31.44 * * \\
\end{array}$ & $\begin{array}{c}- \\
37.49 * * \\
\end{array}$ \\
\hline $\mathrm{P}_{1} \times \mathrm{P}_{4}$ & 1.72 & 1.72 & $11.20 * *$ & $8.37 * *$ & $8.37 * *$ & $-7.58 * *$ & $-9.79 * *$ & $-9.79 * *$ & $\begin{array}{c}- \\
17.75^{* *}\end{array}$ \\
\hline $\mathrm{P}_{1} \times \mathrm{P}_{5}$ & $\begin{array}{c}- \\
10.76^{* *} \\
\end{array}$ & $\begin{array}{c}- \\
11.25^{* *} \\
\end{array}$ & -2.98 & $\begin{array}{c}- \\
11.13^{* *} \\
\end{array}$ & $\begin{array}{c}- \\
18.84 * * \\
\end{array}$ & $\begin{array}{c}- \\
30.78 * * \\
\end{array}$ & $\begin{array}{c}- \\
12.55^{* *} \\
\end{array}$ & $4.94 * *$ & $-4.32 * *$ \\
\hline $\mathrm{P}_{1} \times \mathrm{P}_{6}$ & -0.34 & -0.88 & $8.35 * *$ & $10.77 *$ & $\begin{array}{c}- \\
52.19 * * \\
\end{array}$ & $\begin{array}{c}- \\
59.23^{* *} \\
\end{array}$ & -5.52 & $\begin{array}{c}- \\
40.05 * * \\
\end{array}$ & $45.34 * *$ \\
\hline $\mathrm{P}_{1} \times \mathrm{P}_{7}$ & $-6.88 * *$ & $-7.39 * *$ & 1.24 & $5.01 * *$ & $23.13 * *$ & $5.01 * *$ & $\begin{array}{c}- \\
10.57 * * \\
\end{array}$ & -1.91 & $\begin{array}{c}- \\
10.57 * * \\
\end{array}$ \\
\hline $\mathrm{P}_{1} \times \mathrm{P}_{8}$ & -3.04 & -3.57 & $5.42 *$ & $\begin{array}{c}- \\
13.22 * *\end{array}$ & $\begin{array}{c}- \\
41.48^{* * *} \\
\end{array}$ & $\begin{array}{c}- \\
50.09 * *\end{array}$ & $\begin{array}{c}- \\
23.89 * * \\
\end{array}$ & $\begin{array}{c}- \\
40.89 * * \\
\end{array}$ & $\begin{array}{c}- \\
46.10^{* *} \\
\end{array}$ \\
\hline $\mathrm{P}_{2} \times \mathrm{P}_{3}$ & $-6.01 * *$ & $\begin{array}{c}- \\
11.34 * *\end{array}$ & -3.07 & 3.12 & $\begin{array}{c}- \\
41.67 * * \\
\end{array}$ & $\begin{array}{c}- \\
50.25^{* *}\end{array}$ & -2.94 & $\begin{array}{c}- \\
20.89 * *\end{array}$ & $\begin{array}{c}- \\
27.87 * *\end{array}$ \\
\hline $\mathrm{P}_{2} \times \mathrm{P}_{4}$ & 0.08 & 0.08 & $9.41 * *$ & $8.81 * *$ & $8.81 * *$ & $-7.21 * *$ & $6.16^{* *}$ & $6.16^{* *}$ & $-3.20 * *$ \\
\hline $\mathrm{P}_{2} \times \mathrm{P}_{5}$ & -0.89 & $-6.51 * *$ & 2.20 & $\begin{array}{c}- \\
10.95^{* *}\end{array}$ & $\begin{array}{c}- \\
18.67 * * \\
\end{array}$ & $\begin{array}{c}- \\
30.64 * * \\
\end{array}$ & $\begin{array}{c}- \\
26.64 * * \\
\end{array}$ & $\begin{array}{c}- \\
11.97 * * \\
\end{array}$ & $\begin{array}{c}- \\
19.74 * *\end{array}$ \\
\hline $\mathrm{P}_{2} \times \mathrm{P}_{6}$ & $-7.34 * *$ & $\begin{array}{c}- \\
12.59 * *\end{array}$ & -4.45 & 2.31 & $\begin{array}{c}- \\
42.13^{* *}\end{array}$ & $\begin{array}{c}- \\
50.65 * *\end{array}$ & $-7.80 * *$ & $24.85^{* *}$ & $\begin{array}{c}- \\
31.48 * *\end{array}$ \\
\hline $\mathrm{P}_{2} \times \mathrm{P}_{7}$ & 3.43 & -2.43 & $6.65 * *$ & $4.50 * *$ & $22.53 * *$ & $4.50 * *$ & $7.54 * *$ & $17.95 * *$ & $7.54 * *$ \\
\hline $\mathrm{P}_{2} \times \mathrm{P}_{8}$ & $-5.34 *$ & $\begin{array}{c}- \\
10.71 * * \\
\end{array}$ & -2.39 & $\begin{array}{c}- \\
17.25^{* *} \\
\end{array}$ & $\begin{array}{c}- \\
44.20 * *\end{array}$ & $\begin{array}{c}- \\
52.41 * *\end{array}$ & $\begin{array}{c}- \\
28.15^{* *} \\
\end{array}$ & $\begin{array}{c}- \\
41.43 * * \\
\end{array}$ & $\begin{array}{c}- \\
46.60 * * \\
\end{array}$ \\
\hline $\mathrm{P}_{3} \times \mathrm{P}_{4}$ & 2.98 & 2.98 & $12.57 * *$ & $-6.58 * *$ & $-6.58 * *$ & $\begin{array}{c}- \\
20.33 * * \\
\end{array}$ & $\begin{array}{c}- \\
14.73 * * \\
\end{array}$ & $\begin{array}{c}- \\
14.73 * * \\
\end{array}$ & $\begin{array}{c}- \\
22.25 * * \\
\end{array}$ \\
\hline $\mathrm{P}_{3} \times \mathrm{P}_{5}$ & $-5.65^{*}$ & $\begin{array}{c}- \\
11.00 * * \\
\end{array}$ & -2.71 & $\begin{array}{c}- \\
23.96^{* *}\end{array}$ & $\begin{array}{c}- \\
30.55^{* *} \\
\end{array}$ & $\begin{array}{c}- \\
40.77 * * \\
\end{array}$ & $\begin{array}{c}- \\
36.95 * *\end{array}$ & $\begin{array}{c}- \\
24.34 * *\end{array}$ & $\begin{array}{c}- \\
31.02 * *\end{array}$ \\
\hline $\mathrm{P}_{3} \times \mathrm{P}_{6}$ & $-9.21 * *$ & $\begin{array}{c}- \\
14.36^{* *} \\
\end{array}$ & $-6.38 * *$ & 3.02 & $\begin{array}{c}- \\
49.04 * * \\
\end{array}$ & $\begin{array}{c}- \\
56.54 * * \\
\end{array}$ & $7.68 * *$ & $\begin{array}{c}- \\
31.67 * * \\
\end{array}$ & $\begin{array}{c}- \\
37.70 * * \\
\end{array}$ \\
\hline $\mathrm{P}_{3} \times \mathrm{P}_{7}$ & $-8.41 * *$ & $\begin{array}{c}- \\
13.60 * *\end{array}$ & $-5.55^{*}$ & $4.27 * *$ & $22.26 * *$ & $4.27 * *$ & 0.33 & $10.05 * *$ & 0.33 \\
\hline $\mathrm{P}_{3} \times \mathrm{P}_{8}$ & -3.47 & $-8.94 * *$ & -0.46 & -1.17 & $\begin{array}{c}- \\
33.35^{* *} \\
\end{array}$ & $\begin{array}{c}- \\
43.16^{* *} \\
\end{array}$ & $\begin{array}{c}- \\
20.35^{* *} \\
\end{array}$ & $\begin{array}{c}- \\
38.14 * * \\
\end{array}$ & $\begin{array}{c}- \\
43.60 * * \\
\end{array}$ \\
\hline $\mathrm{P}_{4} \times \mathrm{P}_{5}$ & $7.89 * *$ & $7.89 * *$ & $17.94 * *$ & $18.29 * *$ & $18.29 * *$ & 0.88 & $\begin{array}{c}- \\
13.57 * * \\
\end{array}$ & $3.72 *$ & $-5.43 * *$ \\
\hline $\mathrm{P}_{4} \times \mathrm{P}_{6}$ & $-5.37 *$ & $-5.37 *$ & 3.44 & $\begin{array}{c}- \\
17.67 * * \\
\end{array}$ & $\begin{array}{c}- \\
17.67 * * \\
\end{array}$ & $\begin{array}{c}- \\
29.79 * * \\
\end{array}$ & $\begin{array}{c}- \\
21.52 * * \\
\end{array}$ & $\begin{array}{c}- \\
21.52 * * \\
\end{array}$ & $\begin{array}{c}- \\
28.44 * * \\
\end{array}$ \\
\hline $\mathrm{P}_{4} \times \mathrm{P}_{7}$ & 2.90 & 2.90 & $12.48 * *$ & $37.13 * *$ & $60.80 * *$ & $37.13 * *$ & $23.62 * *$ & $35.58 * *$ & $23.62 * *$ \\
\hline $\mathrm{P}_{4} \times \mathrm{P}_{8}$ & $\begin{array}{c}- \\
10.33^{* *} \\
\end{array}$ & $\begin{array}{c}- \\
10.33^{* *} \\
\end{array}$ & -1.97 & $\begin{array}{c}- \\
20.39 * * \\
\end{array}$ & $\begin{array}{c}- \\
20.39 * * \\
\end{array}$ & $\begin{array}{c}- \\
32.10^{* *} \\
\end{array}$ & -0.73 & -0.73 & $-9.49 * *$ \\
\hline $\mathrm{P}_{5} \times \mathrm{P}_{6}$ & $6.88 * *$ & $-8.65 * *$ & -0.14 & $\begin{array}{c}- \\
23.54 * * \\
\end{array}$ & $\begin{array}{c}- \\
30.17 * * \\
\end{array}$ & $\begin{array}{c}- \\
40.45^{* *} \\
\end{array}$ & $\begin{array}{c}- \\
35.42 * * \\
\end{array}$ & $\begin{array}{c}- \\
22.50 * * \\
\end{array}$ & -29.34 \\
\hline $\mathrm{P}_{5} \times \mathrm{P}_{7}$ & 1.24 & $-7.39 * *$ & 1.24 & $7.28 * *$ & $25.80 * *$ & $7.28 * *$ & $\begin{array}{c}- \\
10.83 * * \\
\end{array}$ & $7.00 * *$ & -2.44 \\
\hline $\mathrm{P}_{5} \times \mathrm{P}_{8}$ & 2.16 & $\begin{array}{c}- \\
12.68 * * \\
\end{array}$ & -4.54 & $\begin{array}{c}- \\
15.98 * * \\
\end{array}$ & $\begin{array}{c}- \\
23.27 * *\end{array}$ & $\begin{array}{c}- \\
34.56 * *\end{array}$ & $\begin{array}{c}- \\
36.78 * *\end{array}$ & $\begin{array}{c}- \\
24.14 * *\end{array}$ & $\begin{array}{c}- \\
30.83 * * \\
\end{array}$ \\
\hline $\mathrm{P}_{6} \times \mathrm{P}_{7}$ & 1.01 & $-7.60 * *$ & 1.01 & $\begin{array}{c}- \\
18.89 * * \\
\end{array}$ & $-4.89 * *$ & $\begin{array}{c}- \\
18.89 * * \\
\end{array}$ & $-7.64 * *$ & 1.29 & $-7.64 * *$ \\
\hline $\mathrm{P}_{6} \times \mathrm{P}_{8}$ & $5.14 *$ & $\begin{array}{c}- \\
11.63 * * \\
\end{array}$ & -3.40 & $43.81 * *$ & -3.02 & $\begin{array}{c}- \\
17.29 * * \\
\end{array}$ & $50.90 * *$ & $17.21 * *$ & $6.86^{* * *}$ \\
\hline $\mathrm{P}_{7} \times \mathrm{P}_{8}$ & -3.95 & $\begin{array}{c}- \\
12.13 * *\end{array}$ & -3.95 & $\begin{array}{c}- \\
25.57 * *\end{array}$ & $\begin{array}{c}- \\
12.72 * *\end{array}$ & $\begin{array}{c}- \\
25.57 * *\end{array}$ & $\begin{array}{c}- \\
11.21 * *\end{array}$ & -2.62 & $\begin{array}{c}- \\
11.21 * *\end{array}$ \\
\hline Mean & -1.99 & -6.43 & 2.29 & -1.30 & -13.86 & -26.54 & -8.82 & -12.01 & -19.97 \\
\hline Highest & 7.89 & 7.89 & 17.94 & 43.81 & 60.80 & 37.13 & 50.90 & 35.58 & 23.62 \\
\hline Lowest & -10.76 & -14.36 & -6.38 & -25.527 & -52.19 & -59.23 & -36.95 & -41.43 & -46.60 \\
\hline
\end{tabular}

* - Significant at 5 per cent probability level, ** - Significant at 1 per cent probability level 
The heterosis for number of fruits per plant varied from $-16.15\left(\mathrm{P}_{7} \times \mathrm{P}_{8}\right)$ to 24.08 per cent $\left(\mathrm{P}_{3} \times \mathrm{P}_{8}\right)$. The heterosis over standard variety $(\mathrm{SV}-1)$ varied from $-26.27\left(\mathrm{P}_{2} \times \mathrm{P}_{3}\right)$ to 23.92 per cent $\left(\mathrm{P}_{1} \times \mathrm{P}_{7}\right)$ and over standard variety $(\mathrm{SV}-2)$ varied from $-31.03\left(\mathrm{P}_{2} \times \mathrm{P}_{3}\right)$ to 15.92 per cent $\left(\mathrm{P}_{1} \times \mathrm{P}_{7}\right)$. Among the 28 crosses, sixteen crosses showed significant values of positive heterosis over better parent while, five crosses showed positive significant heterosis over both standard varieties. Similar observations were also made by Joshi and Thakur (2003), Baishya et al., (2001) and Kumar et al., (2012) with a different set of material in tomato. The extent of heterosis for average fruit weight varied from $-36.95\left(\mathrm{P}_{3} \mathrm{x}\right.$ $\left.\mathrm{P}_{5}\right)$ to 50.90 per cent $\left(\mathrm{P}_{6} \times \mathrm{P}_{8}\right)$. The heterosis over standard variety (SV-1) varied from $41.43\left(\mathrm{P}_{2} \times \mathrm{P}_{8}\right)$ to 35.58 per cent $\left(\mathrm{P}_{4} \times \mathrm{P}_{7}\right)$ and over standard variety $(\mathrm{SV}-2)$ varied $-46.60\left(\mathrm{P}_{2}\right.$ $\left.\mathrm{x} \mathrm{P}_{8}\right)$ to 23.62 per cent $\left(\mathrm{P}_{4} \times \mathrm{P}_{7}\right)$. Out of $28 \mathrm{~F}_{1}$ hybrids, the six crosses over better parent and only three crosses over both the standard varieties showed significant positive heterosis in desirable direction for this trait. These results are in consonance with Sundaram et al., (1994), Baishya et al., (2001) and Garg and Cheema (2010) in tomato. The extent of heterosis for fruit length varied from -10.76 $\left(\mathrm{P}_{1} \times \mathrm{P}_{5}\right)$ to 7.89 per cent $\left(\mathrm{P}_{4} \times \mathrm{P}_{5}\right)$. Heterosis over the standard variety (SV-1) was ranged from -14.36 $\left(\mathrm{P}_{3} \times \mathrm{P}_{6}\right)$ to 7.89 per cent $\left(\mathrm{P}_{4} \mathrm{X}\right.$ $\left.\mathrm{P}_{5}\right)$ and heterosis over the standard variety $(\mathrm{SV}-2)$ ranged from $-6.38\left(\mathrm{P}_{3} \times \mathrm{P}_{6}\right)$ to 17.94 per cent $\left(\mathrm{P}_{4} \mathrm{x} \quad \mathrm{P}_{5}\right)$. The desirable and significant heterosis was observed by three crosses over better parent while, only one cross of SV-1 and nine crosses of SV-2 showed positive significant and desirable heterosis over both the standard varieties. Dev et al., (1994) and Chattopadhyay and Paul (2012) also reported significant heterosis for fruit length in tomato. The range of heterosis per cent for pericarp thickness varied from $14.20\left(\mathrm{P}_{1} \times \mathrm{P}_{3}\right)$ to 17.53 per cent $\left(\mathrm{P}_{4} \times \mathrm{P}_{8}\right)$. The heterosis over standard variety (SV-1) varied from $-11.33\left(\mathrm{P}_{3} . \mathrm{P}_{6}\right)$ to 19.46 per cent $\left(\begin{array}{llll}\mathrm{P}_{5} & \mathrm{x}_{7} & \mathrm{P}_{7} & \text { and over standard variety }(\mathrm{SV}-2)\end{array}\right.$ varied $-8.56\left(\mathrm{P}_{3} \times \mathrm{P}_{6}\right)$ to 23.18 per cent $\left(\mathrm{P}_{5} \mathrm{X}\right.$ $\mathrm{P}_{7}$ ). Out of $28 \mathrm{~F}_{1}$ hybrids, the three crosses over better parent and eleven and fourteen crosses over both the standard varieties showed significant positive heterosis in desirable direction for this trait. The range of heterosis for number of locules per plant in per cent varied from -29.43 $\left(\mathrm{P}_{3} \times \mathrm{P}_{4}\right)$ to 35.26 per cent $\left(\mathrm{P}_{3} \times \mathrm{P}_{6}\right)$. The heterosis over standard variety $(\mathrm{SV}-1)$ varied from $-40.78\left(\mathrm{P}_{2} \times \mathrm{P}_{8}\right)$ to $-3.10\left(\mathrm{P}_{4} \times \mathrm{P}_{5}\right)$ and over standard variety (SV2) varied from $-17.12\left(P_{2} \times P_{8}\right)$ to 35.62 per cent $\left(\mathrm{P}_{4} \times \mathrm{P}_{5}\right)$. Out of $28 \mathrm{~F}_{1}$ hybrids, the seven crosses over better parent and thirteen crosses over only the standard varieties (SV-2) showed significant positive heterosis in desirable direction for this trait. The range of heterosis per cent for total soluble solids varied from $-15.44\left(\mathrm{P}_{4} \times \mathrm{P}_{6}\right)$ to 7.45 per cent $\left(\mathrm{P}_{5} \times \mathrm{P}_{7}\right)$. The heterosis over standard variety $(\mathrm{SV}-1)$ varied from $-33.93\left(\mathrm{P}_{3} \times \mathrm{P}_{5}\right)$ to 2.47 per cent $\left(\mathrm{P}_{4} \times \mathrm{P}_{7}\right)$ and over standard variety $(\mathrm{SV}-2)$ varied from $-8.97\left(\mathrm{P}_{3} \times \mathrm{P}_{5}\right)$ to 41.17 per cent $\left(\mathrm{P}_{4} \times \mathrm{P}_{7}\right)$. Out of $28 \mathrm{~F}_{1}$ crosses, seven crosses over better parent showed only positive desirable heterosis (Table 2).

For fruit circumference, range of heterosis varied from $-15.85\left(\mathrm{P}_{2} \times \mathrm{P}_{3}\right)$ to 14.60 per cent $\left(\mathrm{P}_{7} \times \mathrm{P}_{8}\right)$. The heterosis over standard variety $(\mathrm{SV}-1)$ varied from $-18.50\left(\mathrm{P}_{1} \times \mathrm{P}_{3}\right)$ to 9.80 per cent $\left(\mathrm{P}_{4} \times \mathrm{P}_{7}\right)$ and over standard variety $(\mathrm{SV}-2)$ varied $-6.99\left(\mathrm{P}_{1} \times \mathrm{P}_{3}\right)$ to 25.30 per cent $\left(\mathrm{P}_{4} \times \mathrm{P}_{7}\right)$. Out of $28 \mathrm{~F}_{1}$ crosses, significant positive and desirable heterosis showed by twelve crosses over better parent while, the crosses of standard varieties with the significant and desirable heterosis over the standard were $\mathrm{P}_{4} \times \mathrm{P}_{7}$ and $\mathrm{P}_{2} \times \mathrm{P}_{4}$. Devi et al., (1994) also observed significant positive heterosis for fruit diameter in different cross combination of tomato. With respect to fruit yield per plant the range of heterosis varied from -25.57 $\left(\mathrm{P}_{7} \times \mathrm{P}_{8}\right)$ to 43.81 per cent $\left(\mathrm{P}_{6} \mathrm{x}\right.$ 
$\mathrm{P}_{8}$ ). Heterosis over the standard variety $(\mathrm{SV}$ 1) were from -52.19 $\left(\mathrm{P}_{1} \times \mathrm{P}_{6}\right)$ to 60.80 per cent $\left(\mathrm{P}_{4} \times \mathrm{P}_{7}\right)$ and heterosis over the standard variety $(\mathrm{SV}-2)$ ranged from $-59.23\left(\mathrm{P}_{1} \times \mathrm{P}_{6}\right)$ to 37.13 per cent $\left(\mathrm{P}_{4} \times \mathrm{P}_{7}\right)$. The desirable and significant heterosis were observed by eleven crosses over better parent while, eight crosses of SV-1 and five crosses of SV-2 showed positive significant and desirable heterosis over both the standard varieties. High heterosis for yield/plant was also reported by Dudi and Sanwal (2004), Gul et al., (2011) and Ahmad et al., (2011).

In conclusion, the crosses $\mathrm{P}_{4} \times \mathrm{P}_{7}, \mathrm{P}_{5} \times \mathrm{P}_{7}$ and

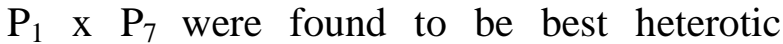
combinations as they exhibited significant heterosis percentage for yield per plant over the standard parent. These high yielding F1 hybrids were expressed 60.80, 25.80 and 23.13 per cent respectively heterosis for yield over standard parent may be recommended for commercial exploitation.

\section{References}

Ahmad, S., Quamruzzaman, A.K.M., and Islam, M.R. 2011. Estimation of heterosis in tomato (Solanum lycopersicum L.). Bangladesh J. Agri. Res., 36(3): 521-527.

Anonymous. 2011. Indian Horticulture Database. NHB, Ministry of Agriculture, Govt. of India.

Baishya, K.C., Syamai, M.M., and Singh, S. P. 2001. Heterosis studies in tomato (Lycopersicon esculentum Mill.). Vegetable Sci., 28(2):168-169.

Chattopadhyay, A., and Paul, A. 2012. Studies on heterosis for different fruit quality parameters in tomato. Int. J. Agri. Environ.
Biotechnol., 5(4): 405-410.

Choudhary, B., Punia, R.S., and Sangha, H.S. 1965. Manifestation of hybrid vigour in F1 and its correlation in F2 generation of tomato (Lycopersicon esculentum Mill). Indian J. Horticulture, 22: 52-59.

Dev, H., Rattan, R.S. and Thakur, M.C. 1994. Heterosis in tomato. The Horticulture J., 7(2): 125-132.

Dubey, R., Das, A., Ojha, M.D., Saha, B., Ranjan, A. and Singh, P.K. 2014. Heterosis and combining ability studies for yield and yield attributing traits in brinjal (Solanum melongena L.). The Bioscan, 9(2): 889-894.

Dudi, B.S., and Sanwal, S.K. 2004.Evaluation for potential F1 hybrids of tomato in respect of fruit yield and components traits. Haryana J. Horticulture Sci., 33(1-2): 98-99.

Gul, R., Rahman, H.U., Khalil, I.H. Shah, S.M.A., and Ghafoor, A. 2011. Estimate of heterosis in tomato (Solanum lycopersicum L.). Bangladesh J. Agri. Res., 36(3): 521-527.

Hedrick, U.P., and Booth, N.O. 1968. Mendelian characters in tomato. Proceedings of American Society Horticulture Science, 5: 19-24.

Joshi, A., and Thakur, M.C. 2003. Exploitation of heterosis for yield and yield contributing traits in tomato (Lycopersicon esculentum Mill.). Progressive Horticulture, 35: 64-68.

Singh, R.K., and Singh, V.K. 1993.Heterosis breeding in tomato (Lycopersicon esculentum Mill.). Annals of Agri. Res., 14: 416-420.

Sundaram, K.S., Irulappan, I., and Thamburaj, S. 1994. Heterosis in two parent, three parent and four parent crosses of tomato (Lycopersicon esculentum Mill.). South Indian Horticulture, 42(5): 309-313.

Yordanov, M. 1983. Heterosis in tomato. Theoretical and Appl. Genetics, 6: 189-219.

\section{How to cite this article:}

Parvendra Kumar, Neeraj Singh, Pradip Kumar Singh. 2017. A Study on Heterosis in Tomato (Solanum lycopersicum L.) for Yield and its Component Traits. Int.J.Curr.Microbiol.App.Sci. 6(7): 1318-1325. doi: https://doi.org/10.20546/ijcmas.2017.607.158 